\title{
The Novartis Vaccines Institute for Global Health: a new initiative for developing vaccines for neglected diseases in developing countries
}

\author{
Allan Saul, Rino Rappuoli. \\ Novartis Vaccines Institute for Global Health S.r.I., Via Fiorentina 1, 53100 Siena, Italy.
}

J Infect Developing Countries 2008; 2(2):154-155.

Received 13 March 2008 - Accepted 17 March 2008.

Copyright (@) 2008 Saul \& Rappuoli. This is an open access article distributed under the Creative Commons Attribution License, which permits unrestricted use, distribution, and reproduction in any medium, provided the original work is properly cited.

In July last year, at the time the first issue of the Journal of Infection in Developing Countries was going to press, a group of infectious disease experts met in Siena, Italy, to consider the direction of another important new initiative in the fight against infectious diseases in developing countries: the Novartis Vaccines Institute for Global Health (NVGH).

The NVGH was created last year (2007) with the not-for-profit mission to develop effective and affordable vaccines for neglected infectious diseases in developing countries. It is a unique concept: an institute created by a large vaccine manufacturer to enable the knowledge and resources within the company to be used to make vaccines that are desperately needed in the developing world, but would not be profitable enough to attract the substantial investment required from commercial sources. To achieve its mission, NVGH will work closely with researchers in developed and developing countries, with public and private funding organizations, and with vaccine manufacturers, especially vaccine manufacturers in developing countries.

The NVGH is now functional with core staff hired, laboratories commissioned, and the first project started. It celebrated its opening in a ceremony and symposium on 22 February 2008. At the symposium, the background and plans for NVGH were presented with talks by Nobel Prize Laureate, Prof. Rolf Zinkernagel on the nature of immunity; Prof. Ogobara Doumbo (University of Bamako, Mali), on the need for vaccines for Africa; Dr. Sam Kariuki, on the epidemiology of drug resistant nontyphoidal salmonella; Prof. Peter
Hotez, on combination vaccine for hookworm and Schistosomiasis; Dr. Roger Glass, on vaccines for rotavirus in developing countries; Prof. Gerd Pluschke, on vaccine for Buruli ulcer; and Prof. Marshall Lightowlers, on vaccine for neurocysticercosis.

In addition to the presentations on the scientific and clinical rational for these vaccines, there were two important talks on how vaccines may be financed. Cardinal Martino described the International Finance Facility for Immunization, and Mr. Monticelli explained the Advanced Market Commitment Scheme for developing and purchasing vaccines for developing countries.

These talks highlighted the two key areas in vaccine development that NVGH will address.

The first is the technical challenge of taking a vaccine from a laboratory concept to a proof of principle in humans. For many neglected diseases, years of patient research have already identified likely candidates for vaccine development. Epidemiologist and clinical researchers have identified populations for testing vaccines. However, the critical missing link is often the production of pilot scale lots of candidate vaccines. This requires production methods to be scaled up; robust formulations to be developed; the vaccines to be produced in GMP conditions then tested for stability; toxicology studies to be performed and regulatory documents to be submitted; and finally the vaccine to be tested for safety.

The knowledge to do the work required in this phase is rarely present in academia, in most biotechs, and in vaccine manufacturers in developing countries. The place where this scarce 
knowledge is present is in a few large vaccine manufacturers that have the experience to conduct this obscure but essential part of the work. Many of the failures in vaccine development are a result of the poor understanding and underestimation of this phase. Attempts at vaccine development in an academic setting are often the beginning of a long agony that requires many years to achieve results that in the appropriate environment would take a few months. The reason is that the academic researchers have to learn ex novo by trial and error what is already validated knowledge in a company that has developed vaccines for decades. Importantly, the quality of the work in this step has a direct impact on the ability of the vaccine to be manufactured after proof of concept has been obtained in humans. There are many Phase 1 and Phase 2 trials of vaccines developed for "neglected" diseases where lack of a properly developed and debugged process makes it difficult to readily transfer the technology to a manufacturer.

NVGH, with direct access to the expertise of Novartis Vaccines, will bring this knowledge and practice to the efficient development of vaccines for neglected infectious diseases.

The second key area that NVGH will address is to ensure that the vaccines that are developed are actually used. This involves a complex set of issues that focus on care in the initial choice and design of vaccines; a combination of controlling the cost of manufacture coupled with funding mechanisms to make the vaccine affordable; and above all, informed health professionals and government officials in target countries who can realistically evaluate the benefits and limits of the vaccine so that they can make appropriate recommendations for inclusion in national health programs.

NVGH aims to maximize the utility of vaccines it helps develop by

- choosing vaccines that have a high potential health benefit and a reasonable feasibility of manufacture.

- ensuring intimate involvement in the development program of researchers and health professionals from counties where a vaccine is likely to be used. We are developing training packages that will involve formal studies leading to postgraduate degrees for younger scientists as well as extensive exchange/sabbatical programs to provide the two-way exchange of information critical for informed decision making in the developing country and at NVGH in Siena. The first MSc program has now commenced with the offer of a fellowship for a student to be undertaken jointly at the Swiss Tropical Institute and at NVGH. This will be the first of many. For updates on the program check www.nvgh.novartis.com.

- ensuring through manufacture, where appropriate, that a country will use the vaccine. This will provide a local market to enhance sustainability of manufacture, but also the incentive to undertake the investment in manufacturing infrastructure and the effort to obtain licensure.

So what was the outcome of the meeting in Siena last July? Following the recommendations of that meeting and further assessment of priorities, NVGH has commenced work on a diarrhoeal disease program. According to World Health Organization figures, diarrhoeal diseases kill more children that HIV, malaria and TB combined; yet with the exception of rotavirus, there are no vaccines suitable for this age group. Work has started on the first vaccine project: a conjugate vaccine that will protect against Salmonella enterica serogroups responsible for typhoid, paratyphoid and nontyphoidal salmonella. This couples the considerable expertise available within Novartis in making conjugate vaccines with a major need, especially for nontyphoidal salmonella in Africa. In addition to the development work, we are putting together the collaborative network that will be able to take this project through to delivery.

If you are interested in being involved either with this project or with projects for vaccines for other neglected diseases, we encourage you to contact us at contact.nvgh@novartis.com.

Corresponding Author: Allan Saul, Novartis Vaccines Institute for Global Health S.r.I., Via Fiorentina 1, 53100 Siena, Italy, email: allan.saul@novartis.com

Conflict of interest: No conflict of interest is declared. 